\title{
Research on the Development of 5G Wireless Communication in the Industrial Field
}

Sen Lin

Jianyan Test Group CO., LTD., Xiamen 363100, Fujian Province, China

\begin{abstract}
The development of science and technology is the key to changing human life and promoting social and economic development. As a product of technological development, the widespread application of communication technology has brought a brand new "dawn" to the development of human society. 5G wireless communication technology is an advanced wireless communication technology that has recently developed. With the advantages of low energy consumption and high network speed, 5G technology has shown very bright development prospects in various fields today. Government, operators and equipment providers are actively promoting and deploying 5G technology, and all links in the industry chain are mature. It is expected that the future market size will reach 17 trillion, especially in today's industrial field, the application of $5 \mathrm{G}$ technology will further enhance work efficiency, ensure work quality, and promote good development in the industrial field. Based on this, this paper will study the development of $5 \mathrm{G}$ wireless technology in the industrial field, so as to provide corresponding reference for the good application of $5 \mathrm{G}$ technology in the industrial field.
\end{abstract}

Keywords: 5G; Wireless communication technology; Industrial field; Industrial development

Publication date: July, 2020

Publication online: 31 July, 2020

*Corresponding author: Sen Lin, 13590470730@163. com

\section{Introduction}

Today is in a period of continuous deepening of the development of the Internet. The Internet is gradually developing from the original interconnection of all people to the interconnection of all things. As the latest mobile communication technology, 5G not only leads today's communication technology to a whole new direction of development, but also makes it reach a commanding height, creating favorable conditions for a new round of global technology and industrial innovation. By applying $5 \mathrm{G}$ to the industrial field, through the construction of " $5 \mathrm{G}+$ Industrial Internet", deep integration between industry and communication technology can be achieved, and a new industrial application mode can be generated to fully meet the needs of industrial intelligence. Therefore, in order to achieve good development in the industrial field, we should focus on the application of $5 \mathrm{G}$ in the industrial field to achieve the perfect fusion of the two.

\section{$25 \mathrm{G}$ wireless communication technology and its development and application status in our country}

\subsection{Introduction to 5G wireless communication technology}

$5 \mathrm{G}$, which is the 5 th generation mobile networks (5th generation mobile networks or 5 th generation wireless systems) is a wireless communication system for the information society services in 2020 , which is an extension and upgrade based on 4G. Compared with 4G, 5G has made a comprehensive upgrade. It has very high capacity and performance, and has very low energy consumption and very low network latency. At the same time, the bandwidth of $5 \mathrm{G}$ is wider, which means that it has a higher transmission rate and can provide users with a better network experience ${ }^{[1]}$. In addition, $5 \mathrm{G}$ can adapt to the high-quality network requirements of many special industries today, and has higher stability and security. 5G technology will usher in explosive mobile data traffic growth and 
massive device connections by providing higher data transmission efficiency, wider service scale, and lower communication delay. More importantly, 5G technology provides technical support for various emerging new business and application scenarios, such as the Internet of Things (IOT), Internet of Vehicles (IOV), Industrial Internet + , big data and broadcast services, as well as lifeline communications in the event of natural disasters. As a result, mobile communication technology has also realized the transformation from personal business have invested in the research and development of $5 \mathrm{G}$ technology.

\subsubsection{Development of 5G communication tech- nology in our country}

In the world, our country is one of the earliest countries to study $5 \mathrm{G}$ technology, which make it the most widely used, and present the best development prospects. The following is the development of $5 \mathrm{G}$ technology in our country:

Table 1. Development of $5 \mathrm{G}$ technology in our country

\begin{tabular}{ccc}
\hline $\mathbf{1}$ & Time & \\
\hline $\mathbf{2}$ & 2017 & Ministry of Industry and Information Technology releases 5G IF spectrum information \\
$\mathbf{3}$ & February, 2018 & Huawei Group successfully completed 5G call test \\
$\mathbf{4}$ & September, 2019 & Huawei successfully developed 5G technology \\
$\mathbf{5}$ & Oct 31, 2019 & 5G commercial packages begin to be announced in our country's three major operators \\
\end{tabular}

applications to industry business applications.

\subsection{Development of $5 \mathrm{G}$ wireless communication technology}

\subsubsection{Development of $5 \mathrm{G}$ communication technol- ogy abroad}

$5 \mathrm{G}$ communication technology is an advanced technology that has been the focus of attention and research in the world in recent years. As early as 2014, the EU began relevant research on 5G technology. At the same time, many developed countries such as France and Germany also carried out this research, and it is expected to put this technology into commercial use. However, because the public does not have a high enough sense of identification with this technology, the development of $5 \mathrm{G}$ technology is restricted. Under such circumstances, countries around the world have not stopped researching

\subsection{Application status of 5 G communication technology}

\subsubsection{Application status of 5G communication technology abroad}

With the development of 5G technology, various countries have begun to attach great importance to the application of $5 \mathrm{G}$ technology. According to the research of MIT scholars, the total number of $5 \mathrm{G}$ users in the United States is expected to be 120 million by the end of 2020, an increase of 50 times compared with 2019. At the same time, 5G technology has also begun to be more and more widely used in other countries, and the application of this technology in different countries is also different. The following Table 2 shows applications of this technology in several developed countries.

Table 2. Application fields of 5G wireless communication technology

\begin{tabular}{ccl}
\hline Serial number & Country & \multicolumn{1}{c}{ Application fields } \\
\hline $\mathbf{1}$ & United States & Wireless broadband, IOV, HD video, VR/AR \\
$\mathbf{2}$ & United Kingdom & Expanding from VR to wireless broadband, IOV and intelligent manufacturing \\
$\mathbf{3}$ & Japan & HD live broadcast, IOV, VR/AR, smart city \\
$\mathbf{4}$ & South Korea & VR/AR, UAV, IOV, security monitoring \\
\hline
\end{tabular}

5G communication technology. According to relevant statistics, as of 2019, 93 countries around the world

2.3.2 Application status of 5G wireless commu- 


\section{nication technology in our country}

Since the application of 5G technology, it has been very well applied in our country. In terms of the application of $5 \mathrm{G}$ technology in China Mobile, as of now, the number of provinces, autonomous regions and municipalities covered by the technology has reached 28 , the number of base stations in demand has reached 232,143, and strives to exceed 500,000 by the end of the year, to ensure the comprehensive popularization of $5 \mathrm{G}$ technology in the business field of prefecture-level cities and above. The following table 3 is the distribution of China Mobile's Phase II wireless main equipment procurement areas and quantities in 2020. to ensure the sending and receiving of minimum number of antennas anytime, anywhere. At the same time, with the increase in the number of antennas, the technology also makes communication more free and enables the reception of diverse information. In the application process of $3 \mathrm{G}$ technology, people have carried out multi-antenna technology inspection with the help of $3 \mathrm{G}$ and WLAN. Through the inspection, it has been found that with the increase of the number of access antennas, the spectrum efficiency in the communication system will also increase, based on this, 5G technology has set up a larger number of access antennas. However, in the

Table 3. Distribution of China Mobile's Phase II wireless main equipment procurement regions and quantities in 2020

\begin{tabular}{cccccc}
\hline Serial number & Area & Quantity & Serial number & Area & Quantity \\
\hline 1 & Guangzhou & 26147 & 15 & Guizhou & 7042 \\
2 & Zhejiang & 22551 & 16 & Heilongjiang & 7012 \\
3 & Jiangsu & 20715 & 17 & Anhui & 6613 \\
4 & Shandong & 19451 & 18 & Liaoning & 6494 \\
5 & Henan & 12402 & 19 & Shanxi & 5888 \\
6 & Sichuan & 10015 & 20 & Hubei & 5051 \\
7 & Yunnan & $9703 \uparrow$ & 21 & Jilin & 3752 \\
8 & Hunan & 9419 & 22 & Neimenggu & 3691 \\
9 & Jiangxi & 8174 & 23 & Gansu & 2858 \\
10 & Shaanxi & 8065 & 24 & Qinghai & 2394 \\
11 & Guangxi & 7594 & 25 & Hainan & 2259 \\
12 & Chongqing & 7339 & 26 & Xizang & 1157 \\
13 & Fujian & 7266 & 27 & Ningxia & 1153 \\
14 & Hebei & 7077 & 28 & Qinghai & 841
\end{tabular}

\section{Key technologies of 5G wireless commu- nication}

\subsection{Multi-input multi-output technology}

This technology is also called MIMO technology, and its main role is to make the system have sufficient spatial grading and multiplexing gains, and achieve significant improvement in wireless communication performance through the significant improvement of spectrum utilization, and to achieve a comprehensive promotion of wireless system capacity and coverage. Today, many people call this technology multi-antenna technology, because it has sufficient channel capacity specific application of this technology, it will also show certain disadvantages. For example, in a single communication system, if the number of antennas is too large, the system space needs to be compressed, and excessive compression of the space will lead to reduction of corresponding performance, which in turn will affect the stability of the system operation ${ }^{[2]}$.

\subsection{D2D technology}

In the past application of wireless communication technology, the center of the networking system is mostly the base station. Through the base station, the network can be covered within a certain range, but this form of networking limits the flexibility of network 
applications to a certain extent. 5G technology can effectively solve this problem. Through the application of D2D technology, not only the burden of base stations can be significantly reduced, but also the limitations of traditional wireless communication will be broken, the delay effect of data transmission is shortened, and the user coverage area is further expanded. The following is a schematic diagram of the structure of a D2D cellular system:

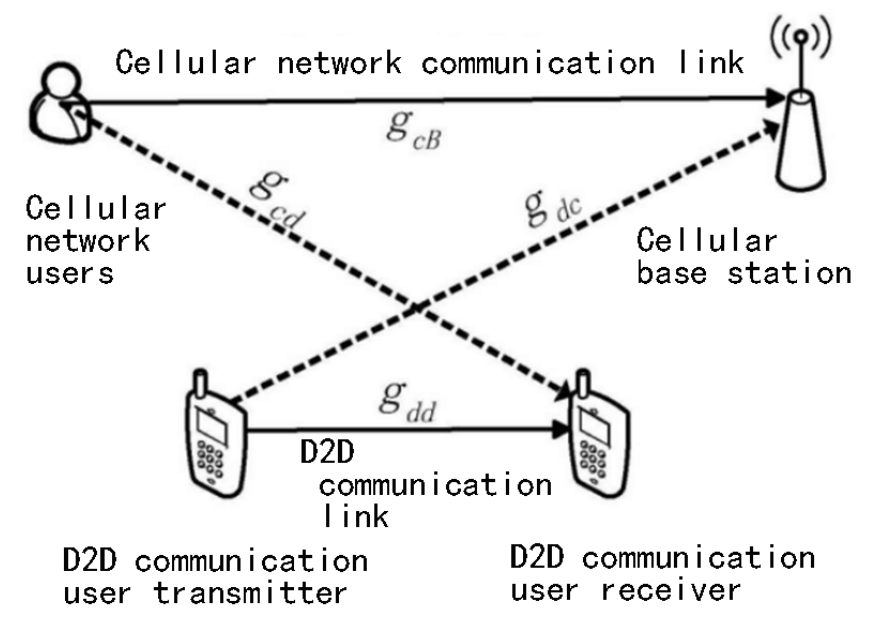

Figure 1. D2D technology cellular system structure diagram

As can be seen from the above figure, with the help of D2D technology, a cellular system can be formed. Data can be transmitted either through the base station or directly between various terminal devices, which makes data transmission between users without the necessity of re-transmission from the base station. In other words, when the surrounding base station fails or stopped working, D2D technology can realize the direct transmission of user-to-user information; When users are within the range where is not covered by wireless signals, they can also communicate through D2D technology, and even achieve multi-user cellular network access.

\subsection{Full-duplex technology}

This technology is a key technology to realize the simultaneous transmission of data information at the same frequency. In the process of data transmission through the traditional wireless communication system, because there is only one channel, no matter the data transmission end or the reception end, there will be a fixed signal interference, so that the transmitting party cannot receive data while transmitting data, and the receiving party cannot transmit data during receiving data. However, due to the full-duplex technology with two independent multiplexed channels, with the help of the full-duplex technology in $5 \mathrm{G}$, information can be transmitted at multiple frequencies to ensure the same-frequency two-way transmission of information. In other words, data can be received and sent at the same time from a workstation. The following is a schematic diagram of data transmission of duplex technology:

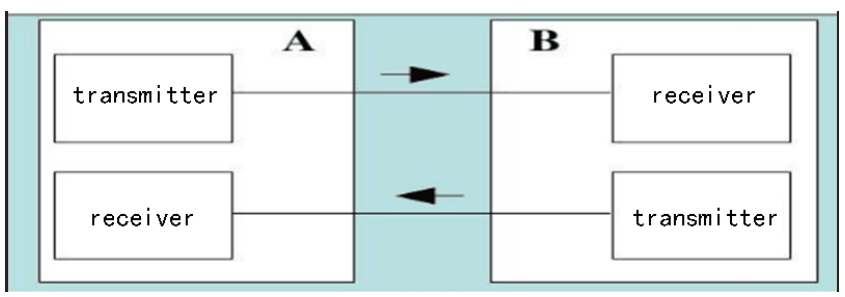

Figure 2. Schematic diagram of data transmission in duplex technology

\section{Development of $5 \mathrm{G}$ wireless commu- nication technology in the industrial field}

\subsection{Realize real-time monitoring and control in the industrial field}

Through 5G technology, real-time monitoring and control of the industrial field can be carried out, so that real-time progress and corresponding problems in the industrial field can be discovered in time, and significant savings in human and material resources in the industrial field can be achieved. At the same time, combining this technology with automatic control and computing technology can comprehensively improve the quality of industrial production and promote well development in the industrial field.

\subsection{Construction of a comprehensive intercon- nection platform}

Because $5 \mathrm{G}$ technology has the characteristics of fast transmission speed, low energy consumption, and can guarantee the original spectrum efficiency, the application of this technology can substantially improve the signal transmission efficiency and transmission quality, and provide highquality and efficient communication support for industrial production. In the process of industrial information transmission through traditional wireless communication technology, it often encounters many problems such as network freeze, failure, packet loss 
or signal interference, which has caused a great deal of trouble for industrial production. Through the application of $5 \mathrm{G}$ technology, the transmission performance in the industrial field can be significantly improved, and a platform that spans equipment, system, factories and regions can be provided for industrial enterprises to achieve comprehensive information interconnection and intercommunication, so as to achieve the industrial production process. The comprehensive optimization of design, manufacturing, service, management, activities, etc. will provide informational support for management innovation and technological innovation in the industrial field $^{[3]}$.

\section{Improve the stability of industrial systems}

The stability of communication technology and network is a key content of industrial system to achieve stable operation. In the past industrial fields, communication networks often have some undesirable conditions in the process of information transmission, which affects the normal operation of industrial systems. In severe cases, the system may even be paralyzed. At the same time, since the occurrence of network information transmission problems are usually sudden and unpredictable, if industrial production enterprises do not take sufficient precautions, once there is a problem in the communication network, it may cause great loss of important information data, industrial production processes may also be abnormal, or even cause production interruptions, such a situation will have a large degree of adverse impact on the economic benefits of industrial enterprises. However, if the 5G technology is reasonably applied to the industrial field, the actual needs of industrial production for the performance of the IOT will be fully met, and then the stability of the industrial system will be comprehensively improved, and the adverse effects of industrial production due to communication network problems will be minimized or avoided.

\section{To achieve precise remote control in the industrial field}

In today's industrial field, automation control technology has been widely used, and the key to automation control technology is remote control. By applying $5 \mathrm{G}$ technology to the industrial field, it is possible to realize precise remote control, thereby realizing significant manpower and time savings in industrial production. For example, in the industrial production process, in order to fully guarantee the accurate and stable data transmission effect between each machine tool, all machine tools can be connected to a terminal in a unified manner, so as to realize the signal connection between the terminal and all machine tools. In the process of connecting through traditional communication technology, a lot of optical cables need to be applied, but due to the large number of optical cables, it will occupy a lot of space and increase the complexity of the entire plant structure ${ }^{[4]}$. At the same time, because the optical cable will have a certain delay in the process of signal transmission, the accuracy of industrial production data information will also be adversely affected to a certain extent. Based on this, if $5 \mathrm{G}$ technology is applied to the industrial field, with its many advantages such as high quality, strong reliability, good stability, and fast transmission speed, it can achieve accurate transmission of AR and high-definition video, etc. On the basis of this, the transmission accuracy is greatly improved to achieve precise remote control in the industrial field.

\section{Conclusion}

In summary, as the most advanced wireless communication technology today, the application of $5 \mathrm{G}$ technology can significantly improve the communication quality and efficiency in the industrial field, it can truly realize the reasonable construction of " $5 \mathrm{G}+$ industrial Internet", solve the existence problems of traditional communication technologies, and comprehensively meet the actual needs of today's industrial production for information transmission. We should believe that with the continuous application and development of $5 \mathrm{G}$ technology in the industrial field, it will play an increasingly significant role in the industrial field, and it will promote the automation and intelligent development of the industrial field.

\section{References:}

[1] Zhu B, Lv CJ. Realization of 5G Network Catalytic Industry 4.0 [J]. Jiangsu Communications, 2020(2): 16-18.

[2] Zhu YD. Research on the necessity of applying 5G to industrial Internet[J]. Science and Technology Innovation, 2020(9): 74-75.

[3] The training of 5G+Industrial Internet in Heilongjiang Station ended successfully[J]. Communication Management and Technology, 2020(2): 13.

[4] Chang SS. Research on Industrial Internet Transformation and Upgrading Based on 5G Empowerment Advantages [J]. Enterprise Reform and Management, 2020(7): 62-63. 